\title{
Cabrera-Mott kinetics of oxidation of metal nanowires
}

\author{
Vladimir P. Zhdanov ${ }^{1,2, a)}$ and Bengt Kasemo ${ }^{1}$ \\ ${ }^{1}$ Department of Applied Physics, Chalmers University of Technology, S-41296 Göteborg, Sweden \\ ${ }^{2}$ Boreskov Institute of Catalysis, Russian Academy of Sciences, Novosibirsk 630090, Russia
}

(Received 1 May 2012; accepted 25 May 2012; published online 13 June 2012)

The Cabrera-Mott model, implying that oxidation of a metal is limited by the field-facilitated activated jumps of metal ions at the metal-oxide interface, was originally proposed to interpret growth of thin oxide films on planar metal surfaces. Recently, the model was used to describe oxidation of spherical nanoparticles with small radius of curvature. Here, we analyze oxidation of nanowires. The increase of the oxide thickness with increasing time for a nanowire is shown to be slower than that for a nanoparticle with the same radius, but faster than in the case of a planar surface. (C) 2012 American Institute of Physics. [http://dx.doi.org/10.1063/1.4729059]

Metal nanowires are currently intensely studied both because of interesting physical phenomena and a variety of applications, ${ }^{1}$ in a similar way as the preceding development with metal nanoparticles. ${ }^{2,3}$ One of the factors limiting their use and/or affecting their functional properties is oxidation. For these reasons, the understanding of the mechanisms and kinetics of oxidation of metals on the nm scale and how they may differ from those of bulk samples is of high interest. As an introductory comment, we recall that the metal oxidation usually starts at the metal-gas or metal-solution interface and then is controlled by diffusion of ions and electrons under the influence of gradients of their concentrations and selfgenerated electric potential. ${ }^{4}$ On the $\mathrm{nm}$ scale, the electric field is often very strong. For macroscopic samples, a generic model describing the kinetics of growth of thin (nm-sized) oxide films under the effect of a strong field was proposed a long time ago by Cabrera and Mott (CM). ${ }^{5}$ Recently, the CM model was modified by us to describe oxidation of metal nanoparticles $^{6}$ (for related interesting molecular dynamics simulations, see Ref. 7). In this letter, we present a similar analysis for metal nanowires.

In the conventional 1D CM model, ${ }^{5}$ molecular or dissociative oxygen adsorption at the oxide surface is considered to be accompanied by the formation of electronic surface states, which in the absence of a field are located above the oxide valence band and below the metal Fermi level, $E_{\mathrm{o}}<E_{\mathrm{F}}$. Electron tunneling from the metal to vacant surface states results in the appearance of charges on the oxide interfaces and accordingly in shift of the energy of these states up to the Fermi energy. The corresponding uniform field generated in the oxide film is $\mathcal{E}=V_{\mathrm{o}} / L \equiv\left(E_{\mathrm{F}}-E_{\mathrm{o}}\right) / e L$, where $L$ is the oxide film thickness and $e$ is the absolute value of the electron charge. The oxide growth is assumed to be limited by activated jumps of metal ions located at the metal-oxide interface (the case of $\mathrm{O}$ ions jumps is treated equivalently). The field is considered to be so strong that the jumps are practically irreversible. The field-induced decrease of the jump activation energy is given by $q b_{0} \mathcal{E}$ or $q b_{0} V_{\mathrm{o}} / L$, where $q$ is the ion charge and $b_{0}$ is the distance between the posi-

${ }^{a)}$ Electronic address: zhdanov@catalysis.ru. tions of the corresponding potential well and barrier. With these ingredients, the growth kinetics is described as

$$
\frac{d L}{d t}=u \exp \left(\frac{A}{L}\right)
$$

where $A \equiv q b_{0} V_{\mathrm{o}} / k_{\mathrm{B}} T$ and $u$ is the field-independent growth constant.

To analyze oxidation of a nanowire, we consider that its shape is cylindrical with the radius $R$ so that the oxide forms a shell of thickness $L$ at $R-L \leq r \leq R$, where $r$ is the radial coordinate. In this model, the charges are uniformly distributed on the surface of cylinders with radii $R$ and $R-L$, and the potential in the oxide is given by $U(r)=-B \ln (r)+C$, where $B$ and $C$ are constants. Taking into account that the shift of the potential in the oxide, $U(R-L)-U(R)$, should be equal to $V_{\mathrm{o}}$, we have $B=V_{\mathrm{o}} / \ln [R /(R-L)]$. The electric field near the metal-oxide interface is accordingly given by

$$
\mathcal{E}=\frac{B}{R-L}=\frac{V_{\mathrm{o}}}{(R-L) \ln [R /(R-L)]} .
$$

The corresponding decrease of the jump activation energy is expressed as $q b_{0} \mathcal{E}$ or $q b_{0} V_{\mathrm{o}} /\{(R-L) \ln [R /(R-L)]\}$. Using the latter expression, we describe the shrinkage of the metal core in analogy with Eq. (1) as

$$
\frac{d \mathcal{R}}{d t}=-u \exp \left(\frac{A}{(R-L) \ln [R /(R-L)]}\right),
$$

where $\mathcal{R} \equiv R-L$ is the core radius.

Due to volume expansion upon oxide formation (this is the most common case), the radius of the whole wire increases. The oxide volume is $2 \pi H\left(R^{2}-\mathcal{R}^{2}\right)$, where $H$ is the wire length. The corresponding volume of the metal before oxidation is $2 \pi H\left(R^{2}-\mathcal{R}^{2}\right) / \eta$, where $\eta>1$ is the expansion coefficient. Adding to the latter volume, the remaining metal volume, $2 \pi H \mathcal{R}^{2}$, we should get the metalwire volume in the absence of oxide, $2 \pi H R_{\mathrm{o}}^{2}$, where $R_{\mathrm{o}}$ is the corresponding wire radius. In this way, we obtain the simplest relation taking volume expansion into account,

$$
\left(R^{2}-\mathcal{R}^{2}\right) / \eta+\mathcal{R}^{2}=R_{\mathrm{o}}^{2}
$$




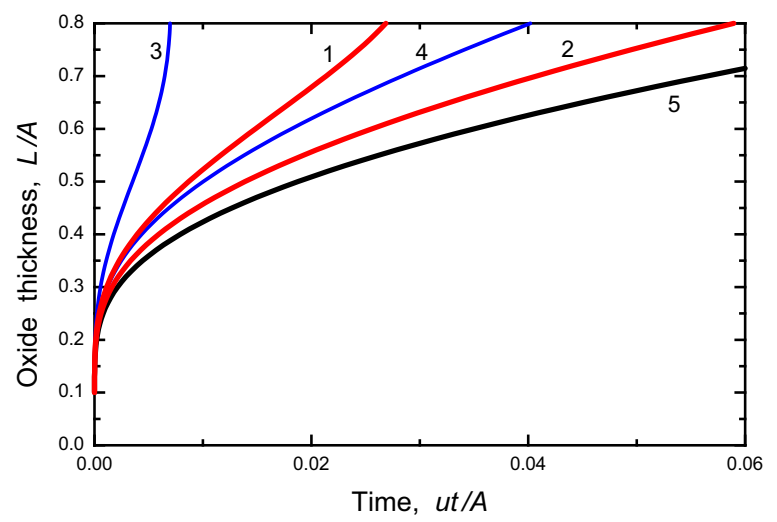

FIG. 1. Oxide thickness as a function of time for $L(0) / A=0.1$. Kinetics 1 and 2 have been calculated for nanowires according to Eq. (5) with $R / A=1$ and 2, respectively. Kinetics 3 and 4 presented for comparison are for spherical nanoparticles according to Eq. (6) with $R / A=1$ and 2, respectively. Kinetics 5 is for a slab according to Eq. (1). (Note that kinetics 3-5 are similar to those shown in Ref. 6 except that here the abscissa is $u t / A$ while in Ref. 6 the abscissa was $50 u t / A$.)

Equations (3) and (4) determine the oxidation kinetics under consideration. In reality, $\eta$ is usually only slightly above unity, and the role of this factor is minor. If we neglect volume expansion and set $\eta=1$, the wire radius will be constant, $R=R_{\mathrm{o}}$ [this physically obvious result follows from Eq. (4)], and $d \mathcal{R} / d t$ will be equal to $-d L / d t$. In this case, Eq. (3) can be rewritten as

$$
\frac{d L}{d t}=u \exp \left(\frac{A}{(R-L) \ln [R /(R-L)]}\right) .
$$

For comparison, we recall that the oxidation of spherical nanoparticles is described as (see Eq. (10) in Ref. 6)

$$
\frac{d L}{d t}=u \exp \left(\frac{A R}{(R-L) L}\right) .
$$

As expected, Eqs. (5) and (6) are identical to Eq. (1) as $R \rightarrow \infty$.

Typical CM kinetics of oxidation of nanowires, spherical nanoparticles, and slabs are shown in Fig. 1. The increase of the thickness of the oxide with increasing time for a nanowire is seen to be slower than for the case of a nanoparticle (provided that their radii are equal) but faster than for the case of a slab.

Finally, we note that nanowires offer advantageous experimental opportunities to track oxidation kinetics and thus to test the theory, because in this case one can use electrical conductivity measurements versus time for different wire radii without oxide and with oxide.

Grant NANO-SEE 181-1 from Swedish Energy Agency is acknowledged.

${ }^{1}$ A. L. Tiano, C. Koenigsmann, A. C. Santulli, and S. S. Wong, Chem. Commun. 46, 8093 (2010).

${ }^{2}$ R. J. White, R. Luque, V. L. Budarin, J. H. Clark, and D. J. MacQuarrie, Chem. Soc. Rev. 38, 481 (2009).

${ }^{3}$ K. M. Mayer and J. H. Hafner, Chem. Rev. 111, 3828 (2011).

${ }^{4}$ E. Fromm, Kinetics of Metal-Gas Interactions at Low Temperatures (Springer, New York, 1998).

${ }^{5}$ N. Cabrera and N. F. Mott, Rep. Prog. Phys. 12, 163 (1948).

${ }^{6}$ V. P. Zhdanov and B. Kasemo, Chem. Phys. Lett. 452, 285 (2008).

${ }^{7}$ B. J. Henz, T. Hawa, and M. R. Zachariah, J. Appl. Phys. 107, 024901 (2010). 